\title{
The Efficacy Between Conventional Lid Hygiene and Additional Thermal Pulsatile System in Meibomian Gland Dysfunction Patients Treated with Long-Term Anti-Glaucoma Medications in a Randomized Controlled Trial
}

This article was published in the following Dove Press journal: Clinical Ophthalmology

\section{Ngamjit Kasetsuwan Disorn Suwajanakorn (D) Chitchanok Tantipat (iD Usanee Reinprayoon}

Department of Ophthalmology, Faculty of Medicine, Chulalongkorn University and King Chulalongkorn Memorial Hospital, Bangkok 10330, Thailand
Correspondence: Chitchanok Tantipat Department of Ophthalmology, Faculty of Medicine, Chulalongkorn University and King Chulalongkorn Memorial Hospital, Bangkok 10330, Thailand

Tel +66-86-III-8524

Fax +66-2-252-8290

Email chitchanok.tob88@gmail.com
Purpose: To evaluate the efficacy of additional thermal pulsatile system compared to standard lid hygiene alone in meibomian gland dysfunction (MGD) patients who are using long-term anti-glaucoma medications.

Patients and Methods: Well-controlled glaucoma patients who used anti-glaucoma medications for at least 1 year and had MGD were enrolled and randomized between a study group who received thermal pulsatile system $\left(\right.$ Lipiflow $^{\mathbb{R}}$ ) treatment and standard lid hygiene, and a control group who received standard lid hygiene alone. The primary outcome was meibomian gland expression score, and the secondary outcomes were the Ocular Surface Disease Index (OSDI) score, Schirmer test score, tear break-up time (TBUT), corneal staining score, lipid layer thickness (LLT), and meibography score. All the outcomes were re-evaluated at 1 week, then at 1,3 , and 6 months after treatment.

Results: Of 60 participants who underwent randomization, 48 completed the study. At the 6-month mark, this study could not demonstrate any significant difference between groups in both primary and secondary outcomes. However, there was significant improvement from baseline in both groups of the following outcomes: meibomian gland expression score, OSDI score, LLT, and meibography score. No serious adverse event was found in this study.

Conclusion: An additional single thermal pulsatile system treatment with standard lid hygiene significantly improved meibomian gland assessment score and subjective symptoms at 6 months. Any difference between additional thermal pulsatile system treatment and lid hygiene alone was not found in this study. The results may suggest more chronic MGD and more damaged meibomian gland induced by long-term anti-glaucoma medications.

Keywords: meibomian gland dysfunction, anti-glaucoma medications, Lipiflow ${ }^{\circledR}$, thermal pulsatile system, lid hygiene

\section{Introduction}

Chronic abnormality of the meibomian glands, together with alteration of gland secretion quality, which lead to tear film instability, are characteristic of meibomian gland dysfunction (MGD). MGD is very common and is the leading cause of evaporative dry eye disease. ${ }^{1}$ Prevalence of MGD varies among geographic regions 
and ethnic groups, but it seems to be highest in the Asian population. ${ }^{1}$ Lekhanont et al revealed that the prevalence of MGD in Bangkok was as high as $46.2 \%{ }^{2}$

Numerous factors are known to cause alteration of meibomian gland function, such as aging, chronic blepharitis, contact lens wear, and anti-glaucoma medications. ${ }^{1,3-5}$ In a population-based study, glaucoma was associated with dry eye signs ${ }^{5}$ and up to $80 \%$ of glaucoma patients had MGD. ${ }^{6}$ One study from the United States demonstrated that nearly half of all glaucoma patients taking antiglaucoma medications (48.4\%) had OSD symptoms. ${ }^{7}$ Direct toxins from the medications, together with their preservatives, are shown to cause subclinical inflammation of conjunctiva and lead to lid margin abnormalities. ${ }^{8,9}$ Agnifili et al used confocal microscopy to demonstrate decreases in gland volume and density induced by topical anti-glaucoma medications. ${ }^{10}$ Number and type of medications also appear to associate with clinical signs of ocular surface disease. ${ }^{4,9,10}$ Duration for medications used for more than 1 year was shown to affect meibomian gland function and morphology. ${ }^{4,10-12}$

Multiple treatment options have been recommended depending on severity of MGD, but the mainstay treatment is lid hygiene. ${ }^{13}$ However, there are many problems regarding the procedure, such as lack of standardized regimen, inadequate heat used, inconvenience, and its timeconsuming nature, that lead to treatment failure and abandonment.

One of new modalities, the thermal pulsatile system or Lipiflow $^{\circledR}$ (TearScience ${ }^{\circledR}$, Morrisville, NC)is designed to provide direct heat to the surface of the inner eyelids and, at the same time, compress both upper and lower lids. The device is composed of 2 important parts: 1) lid warmer, which is similar to a scleral lens with a diameter of $24 \mathrm{~mm}$, and has temperature sensors and precise heater. The inner side of a scleral lens is created to shield the ocular surface from direct exposure to heat; 2) eye cup, which lies on the eyelids, and contains an inflatable air bladder that compresses the eyelids to create a milking effect. Several studies proved the efficacy of a single treatment with this new device, showing the improvement of subjective symptoms and MGD signs for up to 12 months. $^{14-19}$

Despite high incidence of MGD in glaucoma patients, there is no data reported on treatment effects of lid hygiene and the thermal pulsatile system $\left(\right.$ Lipiflow $^{\circledR}$ ) in patients repetitively exposed to long-term anti-glaucoma medications. Therefore, the aim of this study was to evaluate and compare the efficacy of thermal pulsatile system treatment additional to lid hygiene, versus lid hygiene alone, in MGD patients who had been using anti-glaucoma medications.

\section{Materials and Methods}

This prospective, randomized, observer-blind clinical trial was conducted at King Chulalongkorn Memorial Hospital and performed under the approval of the Institutional Review Board and the tenets of the Declaration of Helsinki. The Thai Clinical Trial Registry (TCTR) number was 20,150,919,001. A total of 60 patients were enrolled and participated in the study between October 2015 and November 2016.

Inclusion criteria were as follows: older than 18 years old; used one or more topical anti-glaucoma medications in both eyes for more than 1 year; had MGD stage II or stage III. ${ }^{13}$

Exclusion criteria were as follows: poor vision in one or both eyes; had undergone trabeculotomy or GDD implantation; had advanced glaucoma or uncontrolled IOP; had history of ocular surgery or injury within prior 3 months; had history of ocular herpes within prior 3 months; had active ocular inflammation or infection; had eyelid or ocular surface abnormalities; diagnosed with systemic disease causing dry eyes.

After informed consent was obtained and baseline data were collected, patients were allocated to either the study group or the control group by block randomization, concealed to the investigator in an envelope. All patients were instructed to perform lid hygiene procedure by video demonstration. Then, patients in the study group received an additional 12-minute treatment with Lipiflow ${ }^{\circledR}$, which was done by another physician during the first visit. Reexamination was done at 1 week, then 1,3 , and 6 months after the first visit. All measurements were done only in each patient's right eye by a single, masked investigator.

\section{Lid Hygiene}

To standardize lid hygiene in this study, all patients were provided with a video demonstration. The procedure began with application of a warm towel to compress the eyes for 5 minutes. Then, lid massage was done by applying pressure with a finger toward lid margins. We recommended all patients use baby shampoo in this step. ${ }^{20,21}$ Finally, they were instructed to wash their lids with clean water to remove debris and dry with a clean towel. We also instructed them to perform this procedure at least twice daily while participating in the study. 


\section{Thermal Pulsatile System (Lipiflow ${ }^{\circledR}$ )}

Prior to the insertion of thermal pulsatile system, each patient was instructed to lie down in the semi-supine position and a few drops of topical anesthesia were instilled. The device was inserted in the upper lid first by asking the patient to look down, and vice versa for the lower lid. Both eyes were treated simultaneously for 12 minutes; meanwhile, the patient was advised to close his/ her eyes. After the treatment, the device was removed and the ocular part was disposed of. Topical antibiotics were instilled in both eyes.

\section{Outcome Measurements}

The primary outcome measure was meibomian gland expression score using the meibomian gland evaluator (TearScience ${ }^{\circledR}$, Morrisville, NC). The evaluator was pressed against a total of 15 meibomian glands, each of the 5 glands located in the temporal, central, and nasal lower lid margin. ${ }^{18}$ The gland secretion was graded on a scale from 0 to 3 ( 3 = clear liquid secretion, 2 = cloudy liquid secretion, $1=$ inspissated/toothpaste consistency, and $0=$ no secretion). A total score of 0 to 45 was recorded.

The secondary outcome measures were subjective symptoms, tear break-up time (TBUT), Schirmer test score, corneal staining score, meibography score, and tear film lipid layer thickness (LLT). Dry eye symptoms were assessed using OSDI questionnaire. The total OSDI score ranged from 0 (no symptoms) to 100 (more severe symptoms). Tear break-up time was recorded with an average of 3 measurements using a stopwatch. The Schirmer test score, performed with local anesthesia, was recorded in $\mathrm{mm}$. after 5 minutes. Corneal staining score was graded on a fluorescein staining pattern using Oxford grading scheme. The score ranged from 0 (less severe) to 5 (more severe). The meibography score was analyzed using non-contact meibography (Keratograph 5M (Oculus $\left.{ }^{\circledR}\right)$ ). Infrared images of both upper and lower lids were taken and interpreted. The score was graded on the percentage of atrophic meibomian glands, range from 0 to $3(0=0 \%, 1=$ less than $33 \%$, $2=33$ to $67 \%, 3=$ greater than $67 \%$ ). ${ }^{22}$ The tear film lipid layer thickness was measured using LipiView $^{\circledR}$ (TearScience ${ }^{\circledR}$ Inc., Morrisville, NC, USA). The interferometer analyzed and reported the lipid layer thickness in a value of interferometric color unit (ICU) (1 ICU is reflective of $1 \mathrm{~nm} \mathrm{LLT)}$.

Any adverse symptoms and signs from the thermal pulsatile system $\left(\right.$ Lipiflow $^{\circledR}$ ) treatment were assessed during, immediately after, and 1 week after Lipiflow ${ }^{\circledR}$ treatment. Compliance to lid hygiene treatment was evaluated by the frequency of the procedure performed per week.

\section{Statistical Analysis}

To ensure an adequate sample size, we used the results from Lane et al, which reported the efficacy of Lipiflow ${ }^{\circledR}$ treatment by assessing meibomian gland expressibility. ${ }^{18}$ By using the formula to compare mean values between independent subjects, considering $p$-value of 0.05 to be statistical significance and $80 \%$ to be the study power, the calculated sample size was 24 patients per group. Finally, after adjusting for $20 \%$ dropout rate, the sample size was 30 patients per group.

Descriptive analysis was performed for demographic data, which included sex, age, type of glaucoma, number and type of anti-glaucoma medications, duration of medications used, IOP, and baseline characteristics. To analyze longitudinal data with uneven time points, Generalized Estimation Equations (GEE) were used to compare continuous variables: meibomian gland expression score, the OSDI, Schirmer test score, TBUT, LLT, corneal staining score, and meibography score.

\section{Results}

A total of 60 patients were enrolled and randomized to the study group and control group equally. 26 patients in the study group and 22 patients in the control group completed the study at 6 months. The missing 12 patients were withdrawn from the study due to inconvenience or health problems unrelated to the ocular disease. Mean ages of patients were $66.5 \pm 10.77$ years (range 33-81 years) and $70.23 \pm 8.09$ years (range 58-83 years) in the study group and control group respectively.

Primary open-angle glaucoma was the most common diagnosis in this study (53.8\% in study group and $63.6 \%$ in control group). Majority of the patients in both groups had been prescribed with monotherapy, most frequently prostaglandin analogs. The mean duration of medications used was higher in control group (56.91 \pm 49.84 months) compared to the study group ( $40.27 \pm 23.42$ months), $p>0.05$. Baseline characteristics of patients in both groups are shown in Table 1.

At baseline, meibomian gland expression score in the control group was comparable to the study group. At 1 week after treatment, control group showed slightly greater improvement without statistical significance compared to baseline $(2,95 \% \mathrm{CI}-0.53$ to $4.54, p>0.05)$ than 
Table I Baseline Characteristic Data of Study and Control Groups

\begin{tabular}{|c|c|c|c|}
\hline Characteristics & $\begin{array}{l}\text { Study } \\
(n=26)\end{array}$ & $\begin{array}{l}\text { Control } \\
(n=22)\end{array}$ & $p$-value \\
\hline $\begin{array}{l}\text { Gender } \\
\text { Female } \\
\text { Male }\end{array}$ & $\begin{array}{l}\text { II (42.3\%) } \\
\text { I5 (57.7\%) }\end{array}$ & $\begin{array}{l}14(63.6 \%) \\
8(36.4 \%)\end{array}$ & 0.141 \\
\hline Age (years) & $66.5 \pm 10.77$ & $70.23 \pm 8.09$ & 0.189 \\
\hline $\begin{array}{l}\text { Underlying Disease } \\
\text { Diabetic mellitus } \\
\text { Hypertension } \\
\text { Dyslipidemia } \\
\text { Chronic kidney disease } \\
\text { Lacunar infarction } \\
\text { Others }\end{array}$ & $\begin{array}{l}8(30.8 \%) \\
16(61.5 \%) \\
12(46.2 \%) \\
1(3.8 \%) \\
0(0 \%) \\
8(30.8 \%)\end{array}$ & $\begin{array}{l}9(40.9 \%) \\
14(63.6 \%) \\
10(45.5 \%) \\
1(4.5 \%) \\
2(9.1 \%) \\
6(27.3 \%)\end{array}$ & $\begin{array}{l}0.464 \\
0.881 \\
0.961 \\
0.904 \\
0.116 \\
0.791\end{array}$ \\
\hline $\begin{array}{l}\text { Type of Glaucoma } \\
\text { POAG } \\
\text { PACG } \\
\text { NTG } \\
\text { Others }\end{array}$ & $\begin{array}{l}14(53.8 \%) \\
2(7.7 \%) \\
9(34.6 \%) \\
1(3.8 \%)\end{array}$ & $\begin{array}{l}14(63.6 \%) \\
4(18.2 \%) \\
3(13.6 \%) \\
1(4.5 \%)\end{array}$ & $\begin{array}{l}0.565 \\
0.392 \\
0.18 \\
1\end{array}$ \\
\hline $\begin{array}{l}\text { Type of Glaucoma } \\
\text { Medication } \\
\text { BB } \\
\text { AA } \\
\text { PGA } \\
\text { CAI } \\
\text { Fixed combination }\end{array}$ & $\begin{array}{l}9(34.6 \%) \\
5(19.2 \%) \\
13(50 \%) \\
1(3.8 \%) \\
7(26.9 \%)\end{array}$ & $\begin{array}{l}5(22.7 \%) \\
5(22.7 \%) \\
14(63.6 \%) \\
1(4.5 \%) \\
5(22.7 \%)\end{array}$ & $\begin{array}{l}0.526 \\
1 \\
0.393 \\
1 \\
1\end{array}$ \\
\hline $\begin{array}{l}\text { Number of Medications } \\
1 \\
2 \\
3\end{array}$ & $\begin{array}{l}18(69.2 \%) \\
5(19.2 \%) \\
3(11.5 \%)\end{array}$ & $\begin{array}{l}\text { I } 6(72.7 \%) \\
5(22.7 \%) \\
\text { I ( } 4.5 \%)\end{array}$ & $\begin{array}{l}1 \\
1 \\
0.614\end{array}$ \\
\hline $\begin{array}{l}\text { Number of drops/day } \\
\text { Duration of medication } \\
\text { (month) } \\
\text { IOP }(\mathrm{mmHg}) \\
\text { MGE score } \\
\text { OSDI score } \\
\text { TBUT score } \\
\text { Schirmer test score } \\
\text { Corneal staining score } \\
\text { Meibography score } \\
\text { LLT (nanometer) }\end{array}$ & $\begin{array}{l}2.12 \pm 1.34 \\
40.27 \pm 23.42 \\
12.46 \pm 3.66 \\
21.54 \pm 5.78 \\
23.74 \pm 11.83 \\
5.36 \pm 3.72 \\
6.85 \pm 3.41 \\
1.27 \pm 0.83 \\
3.65 \pm 1.41 \\
65.23 \pm 23.06\end{array}$ & $\begin{array}{l}2 \pm 1.15 \\
56.91 \pm 49.86 \\
12.55 \pm 2.39 \\
22.5 \pm 5.93 \\
26.83 \pm 18.21 \\
5.98 \pm 5.36 \\
9.18 \pm 4.88 \\
1 \pm 0.62 \\
3.77 \pm 1.57 \\
59.27 \pm 20.23\end{array}$ & $\begin{array}{l}0.753 \\
0.162 \\
0.927 \\
0.573 \\
0.484 \\
0.636 \\
0.058 \\
0.204 \\
0.784 \\
0.351\end{array}$ \\
\hline
\end{tabular}

Abbreviations: IOP, intraocular pressure; MGE, meibomian gland expression; OSDI, Ocular Surface Disease Index; TBUT, tear break-up time; LLT, lipid layer thickness; POAG, primary open-angle glaucoma; PACG, primary angle closure glaucoma; NTG, normal-tension glaucoma; BB, beta blocker; AA, alpha agonist; PGA, prostaglandin analog; CAl, carbonic anhydrase inhibitor.

study group $(0.91,95 \% \mathrm{CI}-1.41$ to $3.24, p>0.05)$ (Figure 1). However, the difference in mean change between groups was not significant $(-1.09, p=0.535)$. Both groups revealed gradual improvement of meibomian gland expression score over time, and began to show significant improvement compared to baseline at 3 months (2.68, 95\% CI 0.24 to $5.12, p<0.05$ in control group; $3.33,95 \%$ CI 0.9 to $5.75, p<0.05$ in study group) and at 6 months $(2.99,95 \%$ CI 0.33 to $5.65, p<0.05$ in control group; $4.7,95 \%$ CI 2.2 to $7.2, p<0.05$ in study group). There was no significant difference between groups at any time point. Difference of mean change between groups at 6 months was $1.71, p=0.404$

The OSDI score was higher without being statistically significant in the control group at baseline. The scores were significantly improved compared to baseline after 1 week in both groups $(-5.44,95 \%$ CI -7.17 to $-3.71, p<0.05$ in control group; $-4.91,95 \% \mathrm{CI}-7.21$ to $-2.61, p<0.05$ in study group) (Figure 2) and continued toward 6 months $(-11.78,95 \% \mathrm{CI}-13.5$ to $-10.06, p<0.05$ in control group; $-10,95 \% \mathrm{CI}-12.23$ to $-7.78, p<0.05$ in study group). In the study group, a higher mean subjective symptom score was observed at 1 month, but was not significantly different from how it was at 1 week. Although OSDI scores significantly improved from baseline after treatment, there was no significant difference between groups at any time point. Difference of mean change between groups at 6 months was $1.78, p=0.57$.

The average TBUT values at the baseline of both groups were not significantly different $(p=0.636)$. At 6-month period, there was no significant difference from baseline in both groups $(-0.58,95 \% \mathrm{CI}-2.04$ to $0.89, p>$ 0.05 in control group; $-0.3,95 \% \mathrm{CI}-1.54$ to $0.94, p>$ 0.05 in study group) (Figure 3). Difference of mean change between groups in the $6^{\text {th }}$ month was not statistically significant $(p=0.645)$.

At the baseline, the Schirmer test score in the study group was lower than the control group $(9.18 \pm 4.88$ in control group; $6.85 \pm 3.41$ in study group, $p=0.058$ ).

At the $3^{\text {rd }}$ and $6^{\text {th }}$ month marks, the study group's Schirmer test scores decreased from the baseline markedly, which were $-1.49,95 \%$ CI -2.61 to -0.37 , $p<0.05 ;-1.19,95 \%$ CI -2.34 to $-0.04, p<0.05$ respectively (Figure 4). Meanwhile, such scores in the control group had increased from baseline at the 6-month period $(1.3,95 \% \mathrm{CI}-0.22$ to $2.82, p>0.05)$. However, there was no significant difference between groups in the $6^{\text {th }}$ month.

The LLT average score at the baseline did not possess any significant difference between groups $(59.27 \pm 20.23$ in control group; $65.23 \pm 23.06$ in study group, $p=0.351$ ). 


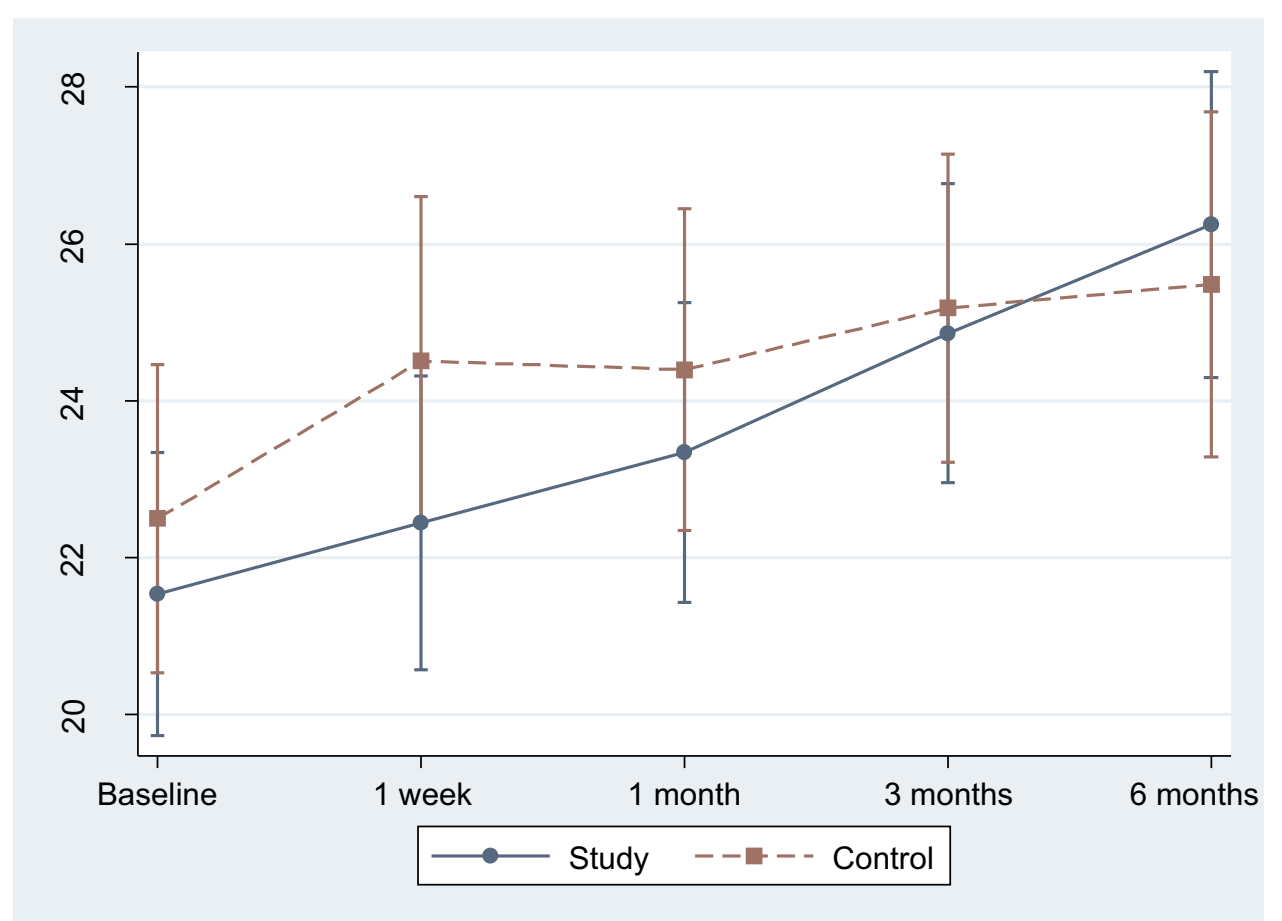

Figure I Mean meibomian gland expression score with standard error at baseline, $1^{\text {st }}$ week, $1^{\text {st }}$ month, $3^{\text {rd }}$ month, and $6^{\text {th }}$ month.

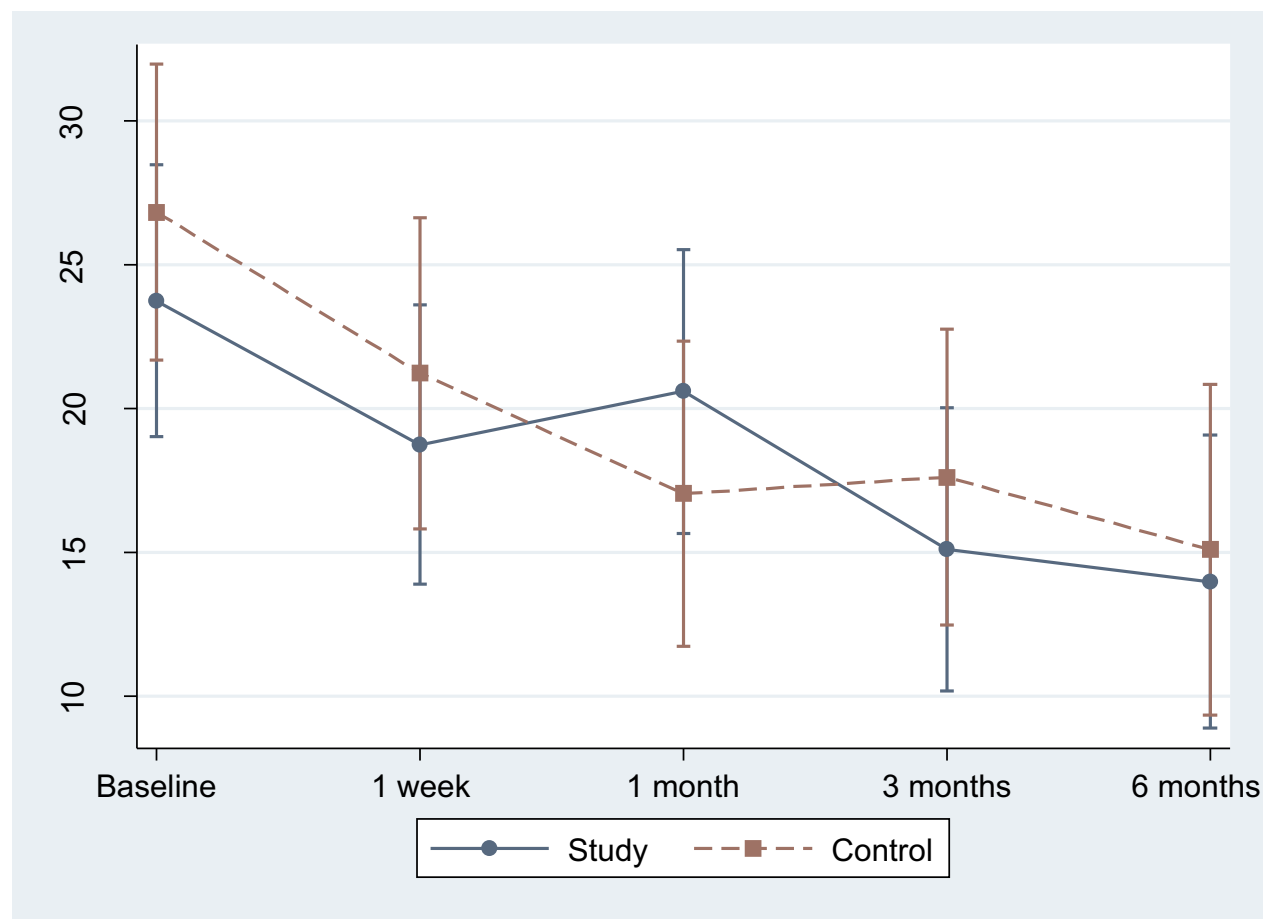

Figure 2 Mean subjective symptoms (OSDI) score with standard error at baseline, $1^{\text {st }}$ week, $1^{\text {st }}$ month, $3^{\text {rd }}$ month, and $6^{\text {th }}$ month.

At the $3^{\text {rd }}$ month mark, LLT score in the control group was found to be increased remarkably $(4.41,95 \%$ CI 1.24 to $7.57, p<0.05$ ) (Figure 5).At 6 months, the LLT score in the study group significantly increased to $2.65,95 \%$ CI
0.08 to $5.22, p<0.05$ but there was no significant difference between groups at this period $(p=0.683)$.

The corneal staining showed no significant difference between groups at the baseline $(1 \pm 0.62$ in control group; 


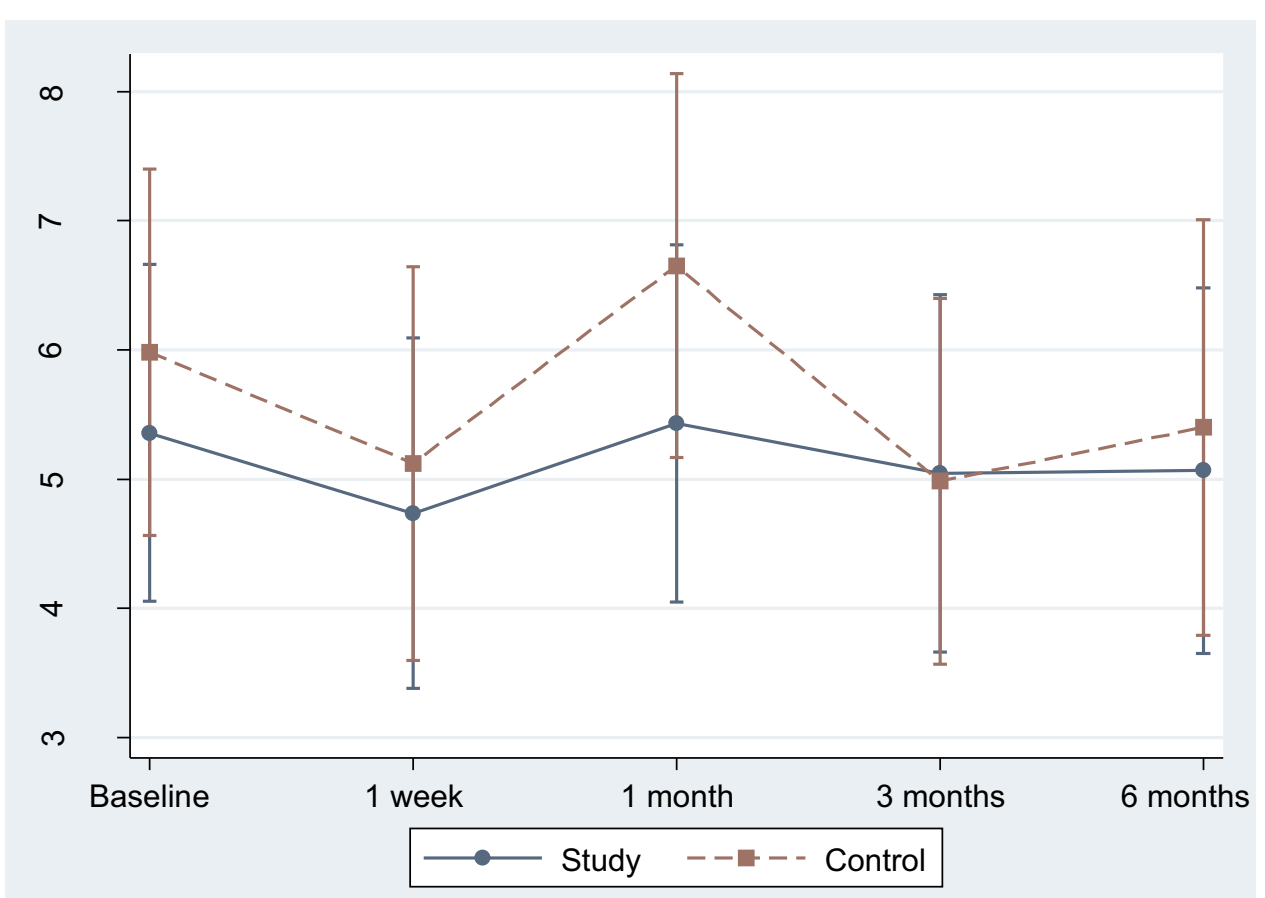

Figure 3 Mean tear break-up time (TBUT) with standard error at baseline, $1^{\text {st }}$ week, $1^{\text {st }}$ month, $3^{\text {rd }}$ month, and $6^{\text {th }}$ month.

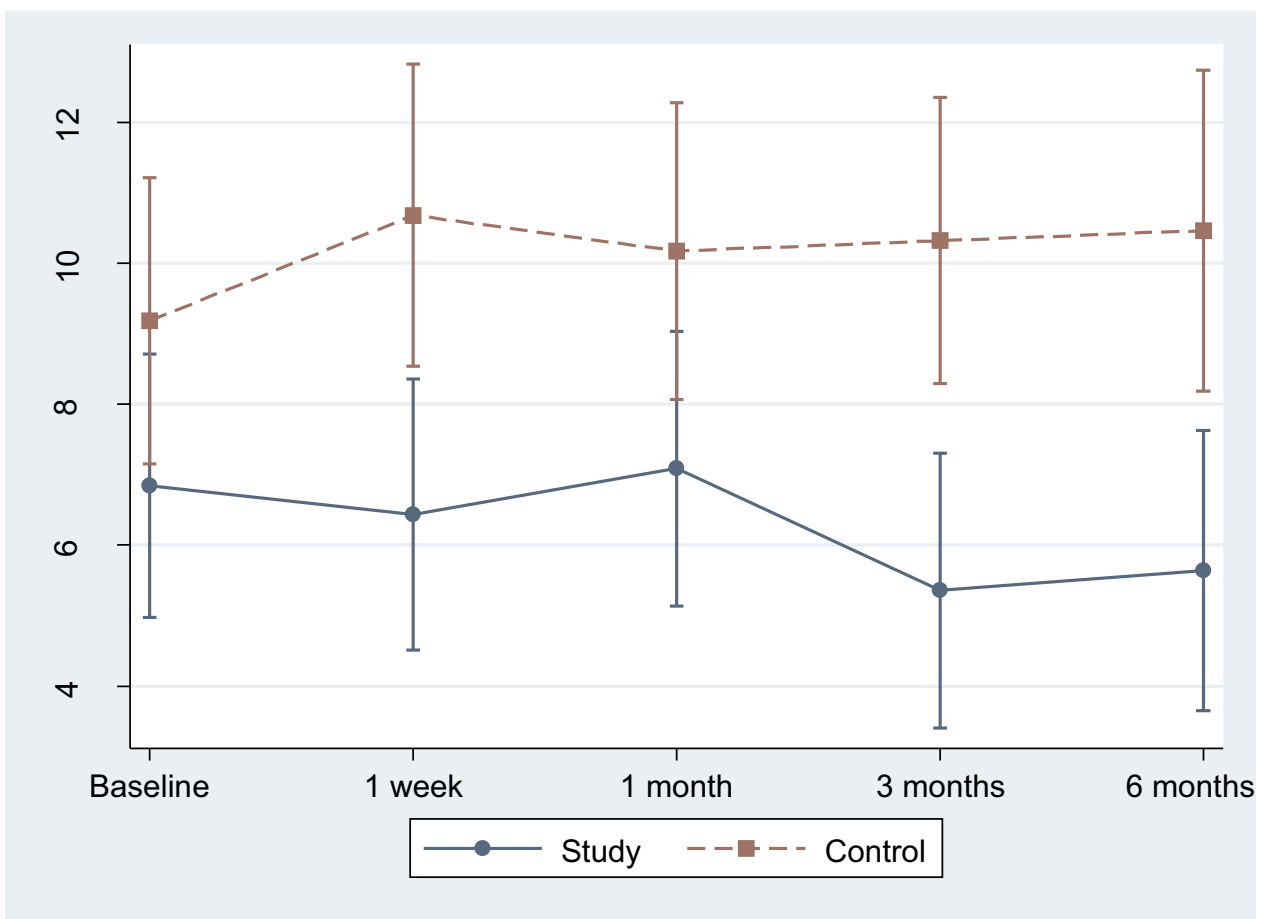

Figure 4 Mean Schirmer test score with standard error at baseline, $I^{\text {st }}$ week, $I^{\text {st }}$ month, $3^{\text {rd }}$ month, and $6^{\text {th }}$ month.

$1.27 \pm 0.83$ in study group, $p>0.05$ ). At 6 months, there was no change between groups from the baseline (Figure 6). There was no significant difference between groups at the $6^{\text {th }}$ month mark $(p=0.488)$.
There was no significant difference in the meibography score between groups at the baseline. Meibography score was found to be decreasing remarkably at 6 months in both groups $(-0.31,95 \% \mathrm{CI}-0.59$ to $0.04, p<0.05$ 


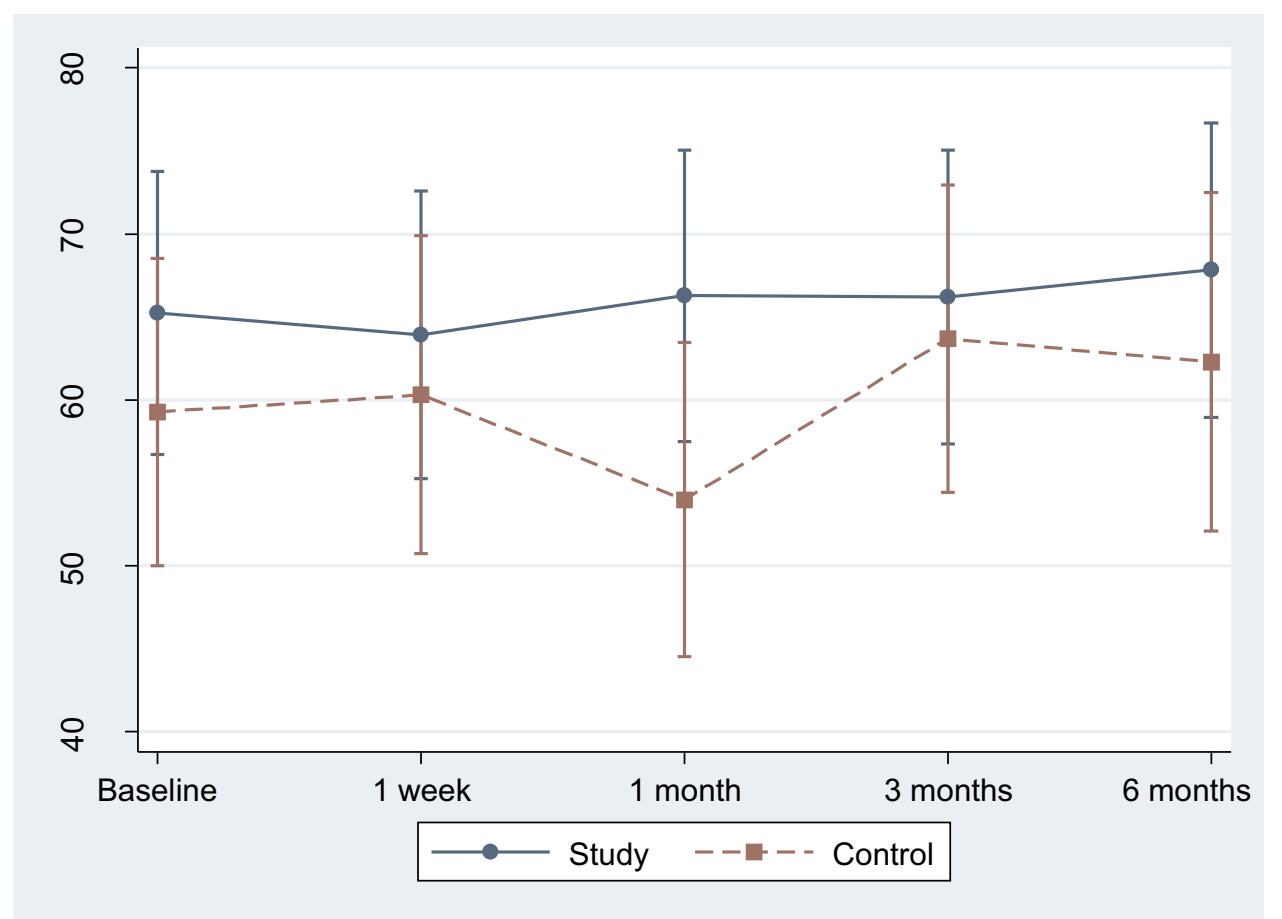

Figure 5 Mean lipid layer thickness (LLT) with standard error at baseline, $1^{\text {st }}$ week, $I^{\text {st }}$ month, $3^{\text {rd }}$ month, and $6^{\text {th }}$ month.

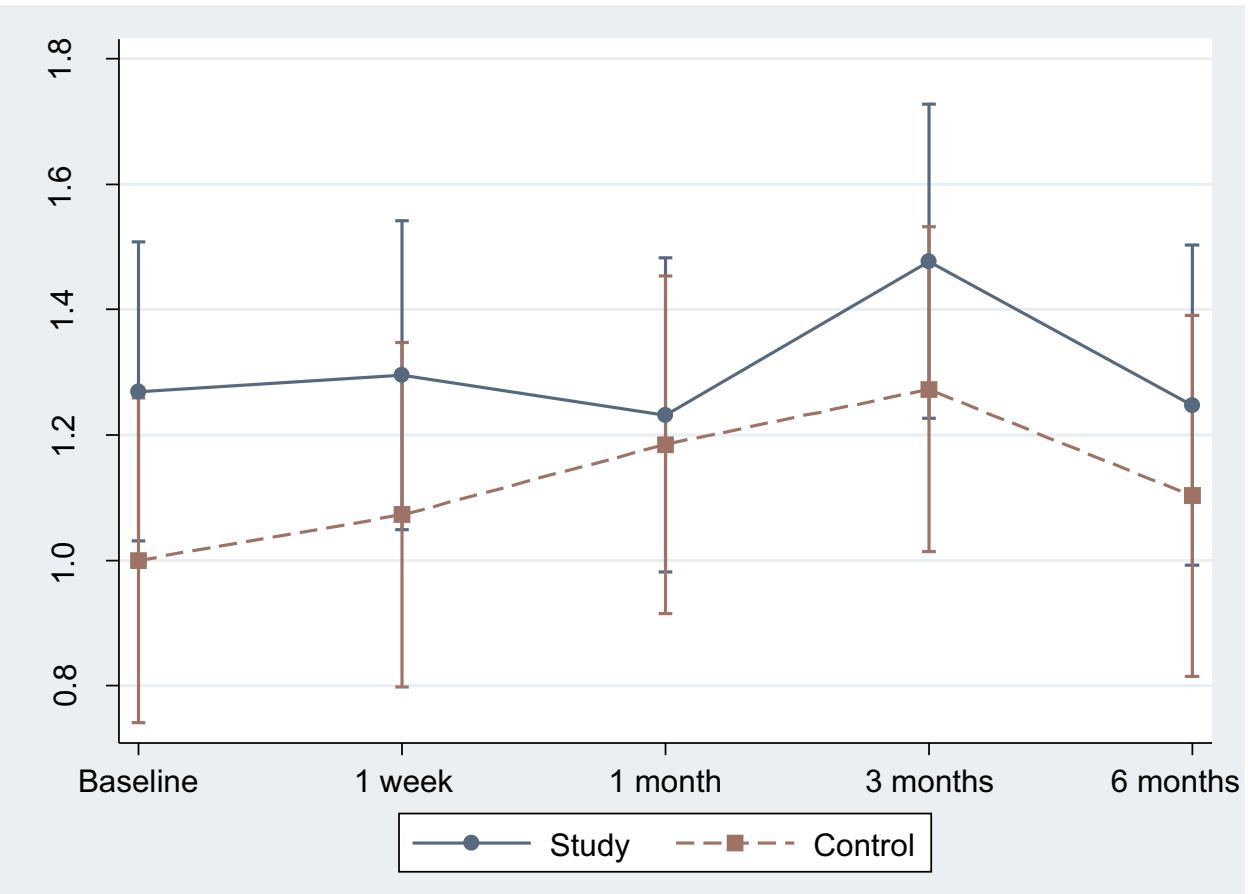

Figure 6 Mean corneal staining score with standard error at baseline, $1^{\text {st }}$ week, $1^{\text {st }}$ month, $3^{\text {rd }}$ month, and $6^{\text {th }}$ month.

in control group; $-0.37,95 \% \mathrm{CI}-0.68$ to -0.05 , In the subgroup analysis according to the systemic $p<0.05$ in study group) (Figure 7). Nevertheless, condition, severity of MGD, types of anti-glaucoma medthere was no significant difference between groups at ication, duration of anti-glaucoma therapy, and the number this period $(0.41, p=0.479)$. of anti-glaucoma medication per day, it was found that the 


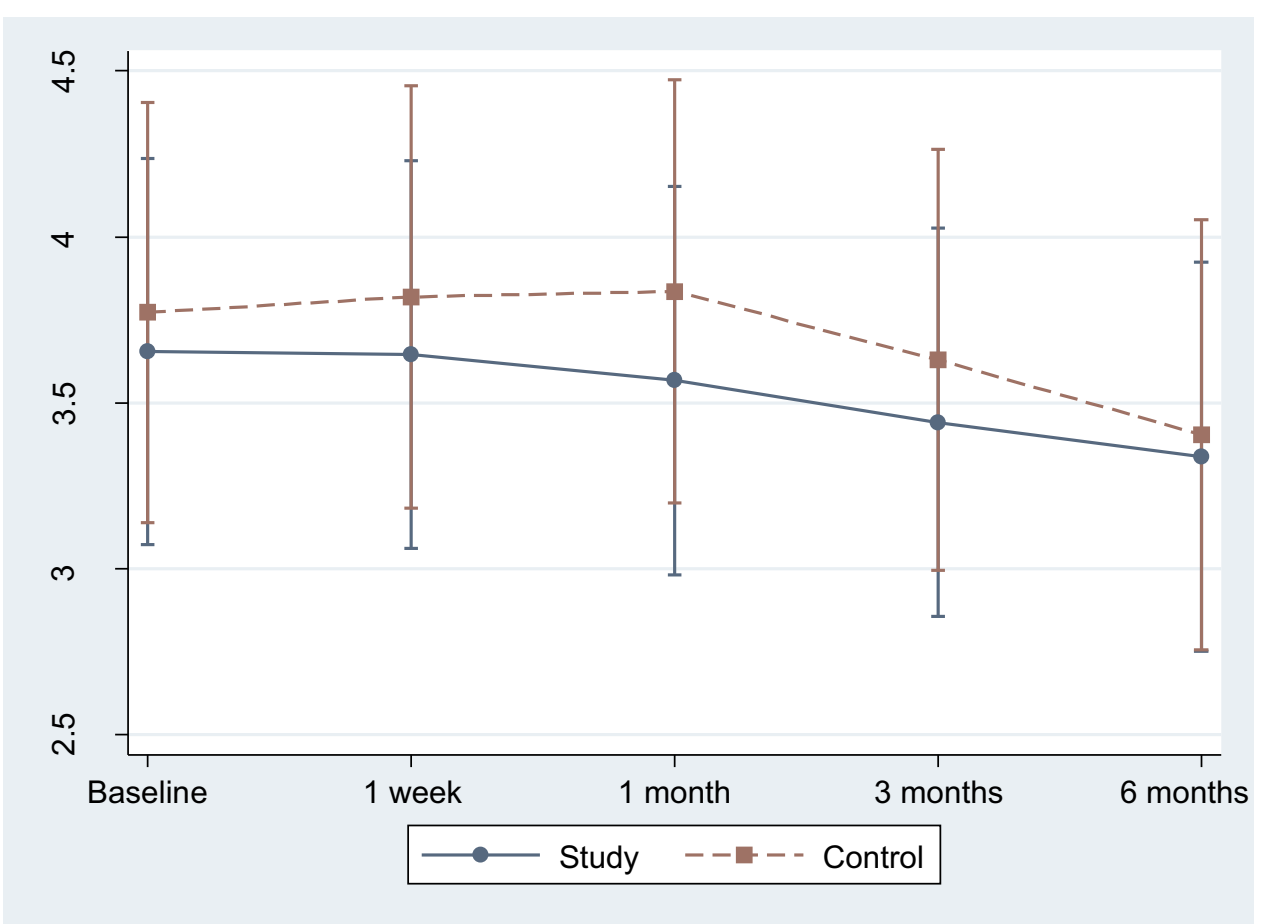

Figure 7 Mean meibography score with standard error at baseline, $1^{\text {st }}$ week, $1^{\text {st }}$ month, $3^{\text {rd }}$ month, and $6^{\text {th }}$ month.

LLT of dyslipidemia (DLP) significantly increased in the $1^{\text {st }}$ month $(-7.14 \pm 2.54$ in control group; $8.27 \pm 2.08$ in study group, $p<0.05$ ) and the LLT of hypertension (HT) significantly increased at the $1^{\text {st }}, 3^{\text {rd }}$, and $6^{\text {th }}$ month in the study group, compared with the control group. At the $6^{\text {th }}$ month marks, the LLT of HT were $-1.56 \pm 2.1$ in control group and $8.83 \pm 1.69$ in study group, $p<0.05$.

According to the severity of MGD, it was found that, in MGD stage II, the LLT $(-9.36 \pm 2.18$ in control group; $2.77 \pm 2.13$ in study group, $p<0.05)$ and TBUT $(-2.01 \pm$ 0.89 in control group; $0.52 \pm 0.9$ in study group, $p<0.05$ ) were increasing in the $1^{\text {st }}$ month. On the other hand, in MGD stage III, the LLT decreased at the $3^{\text {rd }}$ month $(7.6 \pm$ 2.14 in control group; $-1.54 \pm 1.73$ in study group, $p<$ $0.05)$ and the TBUT $(3.88 \pm 1.21$ in control group; $-0.22 \pm$ 0.88 in study group, $p<0.05$ ) decreased at the $1^{\text {st }}$ month in the study group compared with the control group.

In the subgroup analysis according to the duration of anti-glaucoma therapy, the group which had been treated more than 3 years had decreasing OSDI score in the $3^{\text {rd }}$ and $6^{\text {th }}$ month, and the meibography score was reduced in the $1^{\text {st }}$ week, $1^{\text {st }}$ and $3^{\text {rd }}$ month, respectively, in the study group, compared with the control group. Difference of mean change of meibography score between groups at 3 months was $0.58, p<0.05$. Besides the severity of MGD and duration of anti-glaucoma therapy, the subgroup analyses of the types and number of anti-glaucoma medication per day were conducted. Consequently, there was no significant difference between the two groups at every time point.

There was no serious adverse event related to the study treatment. The adverse events found in the study include: one patient had difficulty inserting the device, but after the procedure there was no complication found; five patients reported mild discomfort immediately after the treatment which spontaneously improved in a few hours; one patient reported mild conjunctival injection which resolved in the next few days without further treatment. No IOP elevation was found in any patients or uncontrolled IOP that required additional antiglaucoma treatment in both groups.

Only $12.5 \%$ (6/48) performed lid hygiene at least once a day according to the regimen.Theother 52\% (25/48) performed lid hygiene at least 5 days per week and 23\% (11/ 48) performed an average of 2-3 days per week. 4 patients did not do lid hygiene during the study, despite instruction and encouragement. Most of the reasons for not performing the procedure were inconvenience, forgetfulness and feeling discomfort while performing lid massage.

\section{Discussion}

In this study, the baseline characteristics of both groups did not differ, as shown. However, all participants of this 
study were Thai since MGD is prevalent among the Thai population. This study was also conducted in patients treated with long-term anti-glaucoma medications, hence, the disease might be more severe than in the general population. Such severity might lead to a decrease in an intervention's effect. The aim of this study was to evaluate the efficacy of new modality for MGD treatment, the thermal pulsatile system (Lipiflow ${ }^{\circledR}$ ) in addition to standard lid hygiene, compared to standard lid hygiene alone in patients using long-term anti-glaucoma medications. The study could not demonstrate the difference between study group and control group at 6 months. However, we found significant improvement of meibomian gland expression score, the OSDI score, LLT and meibography score in both groups.

Despite the significant improvement, the change in meibomian gland expression score observed in this study was smaller than in the previous study. Lane et al compared the thermal pulsatile system $\left(\right.$ Lipiflow $\left.^{\circledR}\right)$ with a portable warm compress system or iHeat (Advanced Vision Research, Woburn, MA). ${ }^{18}$ The study reported significant improvement of subjective symptoms, TBUT, meibomian gland assessment in the thermal pulsatile system (Lipiflow ${ }^{\circledR}$ ) group compared with the control group, and also in cross-over group at 2 and 4 weeks. The mean change from baseline to 2 weeks in the thermal pulsatile system (Lipiflow ${ }^{\circledR}$ ) group was 7.9. In an extended study, Greiner re-evaluated the same results at the $9^{\text {th }}$ and $12^{\text {th }}$ month marks and reported significant improvement of mean change from 4.4 to 11.7 and 4.0 to 7.3 , respectively. ${ }^{15,16}$ While in this study, the mean change from baseline to 6 months was 2.99 in control group and 4.7 in study group. The smaller improvement of meibomian gland expression score may result from the shorter follow-up period. Finis et al compared the thermal pulsatile system (Lipiflow ${ }^{\circledR}$ ) and conventional lid hygiene. ${ }^{14}$ At the $3^{\text {rd }}$ month mark, the results showed no significant difference between groups but significant improvement in subjective symptoms, TBUT, tear film LLT, number of expressible meibomian glands, and ocular surface staining score compared to baseline.

The smaller change in the current study may be attributed to more severely diffuse meibomian gland damage induced by repetitive exposure to anti-glaucoma medications and their preservatives. Arita et al showed that using anti-glaucoma medications for more than 1 year affected meibomian gland function and morphology, demonstrating this by comparing ocular surface signs and symptoms in treated eye and contralateral untreated eye. ${ }^{11}$ Other previous studies revealed similar results that dry eye symptoms, lid margin, and tear film abnormalities were higher in patients who used long-term anti-glaucoma medications. ${ }^{6,12}$ The toxicity of preservatives in the topical medication to the ocular surface has been widely demonstrated. Pisella et $\mathrm{al}^{23}$ compared glaucoma patients who used preservative-containing versus preservative-free medication, and found that dry eye symptoms and signs were higher in preservative-containing medication group with dose-dependent effects. Together with the number of drops instilled, the number and duration of medications used were also the risk factors of MGD. ${ }^{4}$

Like Arita et al, ${ }^{12}$ in this study, there was no difference between the two groups when considering the types and number of anti-glaucoma medication. However, when comparing the duration of treatment, it was found that the thermal pulsatile system $\left(\right.$ Lipiflow $^{\circledR}$ ) treatment helped improve the OSDI score and reduced MG loss (meibography score) in the group who had been treated with anti-glaucoma medication more than 3 years. This aligns with Arita et al, who found that MG loss is related to the duration of anti-glaucoma therapy, especially in the group who had been treated with 3 antiglaucoma medications per day.

The mean age of the patients in this study was higher than in the prior study ${ }^{19}$ (66.5 year in study group; 70 year in control group in this study). Aging is proved to be a risk factor for structural and functional alteration of meibomian glands. ${ }^{1}$ Older age is associated with poorer compliance to performing lid hygiene as well. Published data reported moderate to good compliance with lid hygiene. Guillon et $\mathrm{al}^{24}$ found that participants with an average age of 53.5 years tend to have good compliance (average 7 times/week), while Alghamdi et $\mathrm{al}^{21}$ reported moderate compliance $(55 \%)$ in the older participants (mean age of 68.4 years). In contrast to this study, in which although patients were carefully instructed with video demonstration, we found that almost $95 \%$ of patients did not follow the regimen and had moderate to poor compliance. Therefore, this may further explain the higher resistance to MGD treatment in this study. Additional interesting points in terms of compliance are that the frequency was checked and that routine lid hygiene procedures were reviewed at each follow-up examination. The result is stated above. Hence, in real-life practice, it is possible that compliance value would be further decreased, and the thermal pulsatile system $\left(\right.$ Lipiflow $^{\circledR}$ ) usage may help improve treatment results in patients with this problem. 
At the 6-month point, there was no difference between OSDI scores of the study and control group, even though the study group had worse ATD at the baseline and tended to get worse upon follow-up. Intervention for the study group may better aid the improvement of MGD symptoms.

Mean lipid layer thickness (LLT) significantly showed continuous improvement in both groups (4.41 in control group; 2.65 in study group). The smaller improvement might have been due to the mean lipid layer thickness in this study reaching the upper limit for age-matched value, since there is no cut-off point to the normal value of lipid layer thickness, and mean thickness at baseline in this study was equal to mean thickness in normal control group in some studies ${ }^{25,26}(59.27 \pm 20.23$ in control group; $65.23 \pm$ 23.06 in study group). Furthermore, it may be explained by more damage of the meibomian gland being induced by longterm anti-glaucoma medications.

Moreover, according to the graph (Figure 5), the study group's LLT had continuously risen toward the $6^{\text {th }}$ month mark and might increase if followed further. On the other hand, the LLT graph of the control group fluctuated more, which might be the result from other factors, such as the compliance of patients in each period.

Nevertheless, upon conducting subgroup analysis, the LLT increased significantly in the study group of MGD stage II in the $1^{\text {st }}$ month after treatment. This change was not found in MGD stage III and aligns with the study results of Finis et al, ${ }^{27}$ which revealed that the patients in the early stage of MGD would likely benefit from this thermal pulsatile system $\left(\right.$ Lipiflow $^{\circledR}$ ) treatment. Moreover, in the HT and DLP participants, who were diagnosed with associated systemic related factors for MGD ${ }^{28}$ the LLT increased in the study group more than the control group significantly.

Atrophic glands of both groups in the $6^{\text {th }}$ month decreased equally. Nevertheless, there were some differences between 2 graphs (Figure 7). Whereas the study group's atrophic glands had been lowered continuously from the beginning, the control group's rose in the first month and fell gradually, which might be an effect of other factors such as patients' compliance in each period.

Adverse events from the thermal pulsatile system (Lipiflow ${ }^{\circledR}$ ) treatment found in this study were similar to the previous reports. In Lane et al, after the treatment, some patients had conjunctival hyperemia, mild conjunctival injection, and mild petechial hemorrhage, all of which spontaneously resolved. ${ }^{18}$ During the thermal pulsatile system $\left(\right.$ Lipiflow $^{\circledR}$ ) treatment, a patient reported mild discomfort. Mean IOP was slightly increased $(<1 \mathrm{mmHg})$ immediately after treatment and decreased without further treatment afterward. No serious adverse event was found in that study. In this study, no serious adverse event related to the treatment occurred either. The most severe complication found was mild discomfort during or immediately after the treatment. All events resolved without further treatment in a few days. There was no change in IOP after the treatment and no patients required additional antiglaucoma medications. Thus, the thermal pulsatile system $\left(\right.$ Lipiflow $^{\circledR}$ ) is safe for glaucoma patients who have stable IOP and have not receive filtering surgery.

The current study has some limitations. Firstly, even though we enrolled glaucoma patients who used long-term (more than 1 year) anti-glaucoma medications, each patient may have suffered from meibomian gland dysfunction at another time before enrollment. The duration or chronicity of disease itself may affect the results as well. Secondly, MGD is a complex multifactorial disease which has wide variation of symptoms and signs, even in the same stage. The disease lacks a specific reliable sign to indicate the course of the disease. Furthermore, clinical signs may not correlate with subjective symptoms. Thirdly, type, number, and duration of anti-glaucoma medications used are one of many key factors affecting the outcomes. These factors were not scoped or evaluated in this study.

Apart from the patients' old age, their conditions of dry eyes and glaucoma could cause them to be depressed. ${ }^{29}$ This would probably influence their compliance. Thus, Lipiflow treatment between months 6-12 might help improve the treatment outcomes in patients with poor compliance.

Glaucoma patients in this study should have been treated with preservative-free medications. While most patients received only one medication, some patients received many, which might cause disease to be worsened. Therefore, the latter group of patients would be better treated with early surgery or laser treatment.

In the future, there should be a study conducted in a larger-sized sample, and it should be followed up within a longer period of time in order to demonstrate a clearer treatment result in terms of variations such as LLT and meibography score.

\section{Conclusion}

This is the first study to evaluate the effectiveness of the additional thermal pulsatile system compared to standard lid 
hygiene alone in patients using longterm anti-glaucoma medications. The additional single thermal pulsatile system (Lipiflow ${ }^{\circledR}$ ) treatment with standard lid hygiene significantly improved meibomian gland expression score, subjective symptoms, LLT, meibography score at 6 months. No differences between the additional thermal pulsatile system (Lipiflow ${ }^{\circledR}$ ) treatment and lid hygiene alone were found in this study. The results may suggest the problem is more chronic MGD and more damaged meibomian gland is being induced by long-term anti-glaucoma medications. Despite the compliance problem, this study reinforces the importance of lid hygiene for the treatment of MGD.

\section{Data Sharing Statement}

The data that support the findings of this study are available from King Chulalongkorn Memorial Hospital, but restrictions apply to the availability of these data and so are not publicly available. However, data are available from the authors upon reasonable request and with the permission of the institution.

\section{Disclosure}

The authors have indicated that they have no conflicts of interest regarding the content of this article.

\section{References}

1. Schaumberg DA, Nichols JJ, Papas EB, Tong L, Uchino M, Nichols KK. The international workshop on meibomian gland dysfunction: report of the subcommittee on the epidemiology of, and associated risk factors for, MGD. Invest Ophthalmol Vis Sci. 2011;52 (4):1994-2005. doi:10.1167/iovs.10-6997e

2. Lekhanont K, Rojanaporn D, Chuck RS, Vongthongsri A. Prevalence of dry eye in Bangkok, Thailand. Cornea. 2006;25(10):1162-1167. doi:10.1097/01.ico.0000244875.92879.1a

3. Arici MK, Arici DS, Topalkara A, Guler C. Adverse effects of topical antiglaucoma drugs on the ocular surface. Clin Experiment Ophthalmol. 2000;28(2):113-117. doi:10.1046/j.1442-9071.2000. 00237.x

4. Ghosh S, O'Hare F, Lamoureux E, Vajpayee RB, Crowston JG. Prevalence of signs and symptoms of ocular surface disease in individuals treated and not treated with glaucoma medication. Clin Experiment Ophthalmol. 2012;40(7):675-681. doi:10.1111/j.14429071.2012.02781.x

5. Mathews PM, Ramulu PY, Friedman DS, Utine CA, Akpek EK. Evaluation of ocular surface disease in patients with glaucoma Ophthalmology. 2013;120(11):2241-2248. doi:10.1016/j.ophtha.20 13.03.045

6. Uzunosmanoglu E, Mocan MC, Kocabeyoglu S, Karakaya J, Irkec M. Meibomian gland dysfunction in patients receiving long-term glaucoma medications. Cornea. 2016;35(8):1112-1116. doi:10.1097/ ICO.0000000000000838

7. Fechtner RD, Godfrey DG, Budenz D, Stewart JA, Stewart WC, Jasek MC. Prevalence of ocular surface complaints in patients with glaucoma using topical intraocular pressure-lowering medications. Cornea. 2010;29(6):618-621. doi:10.1097/ICO.0b013e3181c325b2
8. Mietz H, Niesen U, Krieglstein GK. The effect of preservatives and antiglaucomatous medication on the histopathology of the conjunctiva. Graefe's arch clin exp ophthalmol/Albrecht von Graefes Archiv fur klinische und experimentelle Ophthalmologie. 1994;232(9):561-565. doi:10.1007/BF00182000

9. Brandt JD, Wittpenn JR, Katz LJ, Steinmann WN, Spaeth GL. Conjunctival impression cytology in patients with glaucoma using long-term topical medication. Am $J$ Ophthalmol. 1991;112 (3):297-301. doi:10.1016/S0002-9394(14)76730-3

10. Agnifili L, Fasanella V, Costagliola C, et al. In vivo confocal microscopy of meibomian glands in glaucoma. Br J Ophthalmol. 2013;97 (3):343-349. doi:10.1136/bjophthalmol-2012-302597

11. Arita R, Itoh K, Maeda S, et al. Effects of long-term topical anti-glaucoma medications on meibomian glands. Graefe's arch clin exp ophthalmol/Albrecht von Graefes Archiv fur klinische und experimentelle Ophthalmologie. 2012;250(8):1181-1185. doi:10.1007/ s00417-012-1943-6

12. Arita R, Itoh K, Maeda S, et al. Comparison of the long-term effects of various topical antiglaucoma medications on meibomian glands. Cornea. 2012;31(11):1229-1234. doi:10.1097/ICO.0b013e31823f8 e7d

13. Geerling G, Tauber J, Baudouin C, et al. The international workshop on meibomian gland dysfunction: report of the subcommittee on management and treatment of meibomian gland dysfunction. Invest Ophthalmol Vis Sci. 2011;52(4):2050-2064. doi:10.1167/iovs.10$6997 \mathrm{~g}$

14. Finis D, Hayajneh J, Konig C, Borrelli M, Schrader S, Geerling G. Evaluation of an automated thermodynamic treatment (LipiFlow ${ }^{\circledR}$ ) system for meibomian gland dysfunction: a prospective, randomized, observer-masked trial. Ocul Surf. 2014;12(2):146-154. doi:10.1016/j. jtos.2013.12.001

15. Greiner JV. A single LipiFlow ${ }^{\circledR}$ thermal pulsation system treatment improves meibomian gland function and reduces dry eye symptoms for 9 months. Curr Eye Res. 2012;37(4):272-278. doi:10.3109/ 02713683.2011.631721

16. Greiner JV. Long-term (12-month) improvement in meibomian gland function and reduced dry eye symptoms with a single thermal pulsation treatment. Clin Experiment Ophthalmol. 2013;41(6):524-530. doi:10.1111/ceo.12033

17. Korb DR, Blackie CA. Case report: a successful LipiFlow ${ }^{\circledR}$ treatment of a single case of meibomian gland dysfunction and dropout. Eye Contact Lens. 2013;39(3):e1-3. doi:10.1097/ICL.0b013e318 $24 \mathrm{ccbda}$

18. Lane SS, DuBiner HB, Epstein RJ, et al. A new system, the LipiFlow $^{\circledR}$, for the treatment of meibomian gland dysfunction. Cornea. 2012;31(4):396-404. doi:10.1097/ICO.0b013e318239aaea

19. Blackie CA, Coleman CA, Holland EJ. The sustained effect (12 months) of a single-dose vectored thermal pulsation procedure for meibomian gland dysfunction and evaporative dry eye. Clin Ophthalmol. 2016;10:1385-1396. doi:10.2147/OPTH.S109663

20. Key JE. A comparative study of eyelid cleaning regimens in chronic blepharitis. CLAO J. 1996;22(3):209-212.

21. Alghamdi YA, Camp A, Feuer W, Karp CL, Wellik S, Galor A. Compliance and subjective patient responses to eyelid hygiene. Eye Contact Lens. 2016.

22. Finis D, Ackermann P, Pischel N, et al. Evaluation of meibomian gland dysfunction and local distribution of meibomian gland atrophy by non-contact infrared meibography. Curr Eye Res. 2015;40 (10):982-989. doi:10.3109/02713683.2014.971929

23. Pisella PJ, Pouliquen P, Baudouin C. Prevalence of ocular symptoms and signs with preserved and preservative free glaucoma medication. Br J Ophthalmol. 2002;86(4):418-423. doi:10.1136/bjo.86.4.418

24. Guillon M, Maissa C, Wong S. Eyelid margin modification associated with eyelid hygiene in anterior blepharitis and meibomian gland dysfunction. Eye Contact Lens. 2012;38(5):319-325. doi:10.1097/ ICL.0b013e318268305a 
25. Eom Y, Lee JS, Kang SY, Kim HM, Song JS. Correlation between quantitative measurements of tear film lipid layer thickness and meibomian gland loss in patients with obstructive meibomian gland dysfunction and normal controls. Am J Ophthalmol. 2013;155 (6):1104-10.e2. doi:10.1016/j.ajo.2013.01.008

26. Zhao Y, Tan CL, Tong L. Intra-observer and inter-observer repeatability of ocular surface interferometer in measuring lipid layer thickness. $B M C$ Ophthalmol. 2015;15:53. doi:10.1186/s12886-015-0036-9

27. Finis D, König C, Hayajneh J, Borrelli M, Schrader S, Geerling G. Six-month effects of a thermodynamic treatment for MGD and implications of meibomian gland atrophy. Cornea. 2014;33 (12):1265-1270. doi:10.1097/ICO.0000000000000273
28. Nichols KK, Foulks GN, Bron AJ, et al. The international workshop on meibomian gland dysfunction: executive summary. Invest Ophthalmol Vis Sci. 2011;52(4):1922-1929. doi:10.1167/iovs.106997a

29. Zheng Y, Wu X, Lin X, Lin H. The prevalence of depression and depressive symptoms among eye disease patients: a systematic review and meta-analysis. Sci Rep. 2017;7:46453. doi:10.1038/ srep46453
Clinical Ophthalmology

\section{Publish your work in this journal}

Clinical Ophthalmology is an international, peer-reviewed journal covering all subspecialties within ophthalmology. Key topics include: Optometry; Visual science; Pharmacology and drug therapy in eye diseases; Basic Sciences; Primary and Secondary eye care; Patient Safety and Quality of Care Improvements. This journal is indexed on PubMed

Submit your manuscript here: https://www.dovepress.com/clinical-ophthalmology-journal
Dovepress

Central and CAS, and is the official journal of The Society of Clinical Ophthalmology (SCO). The manuscript management system is completely online and includes a very quick and fair peer-review system, which is all easy to use. Visit http://www.dovepress.com/ testimonials.php to read real quotes from published authors. 